Rect i fi ed Pr ot on Gr ot thuss Conduct i on Across a Long Wat er-Wre in the Test Nanot ube of the Pol yt heonami de B Channel .

\begin{tabular}{|c|c|}
\hline 著者 & $\begin{array}{l}\text { Nat suki Yuka, I wamto Nasayuki, M ta } \\
\text { Keni chi ro, Shi gemi Kenj i, Nat sunaga Shi geki } \\
\text { Oi ki Shi get oshi }\end{array}$ \\
\hline $\begin{array}{l}\text { j our nal or } \\
\text { publ i cat } i \text { on } t i t l e\end{array}$ & Journal of the Amer i can Chem cal Soci et y \\
\hline vol une & 138 \\
\hline number & 12 \\
\hline page $r$ ange & 4168-4177 \\
\hline year & 2016-03-09 \\
\hline URL & ht t p: //hdl . handl e. net /10098/10179 \\
\hline
\end{tabular}




\title{
Rectified Proton Grotthuss Conduction Across a Long Water- Wire in the Test Nano-Tube of the Polytheonamide B Channel
}

\author{
Yuka Matsuki $^{1,2}$, Masayuki Iwamoto ${ }^{1}$, Kenichiro Mita ${ }^{1,2}$, Kenji Shigemi ${ }^{2}$, Shigeki Matsunaga ${ }^{3}$ and \\ *Shigetoshi Oiki ${ }^{1}$
}

\author{
${ }^{1}$ Department of Molecular Physiology and Biophysics, Faculty of Medical Sciences, University of Fukui, Fukui 910-1193, \\ Japan, \\ ${ }^{2}$ Department of Anesthesiology and Reanimatology, Faculty of Medical Sciences, University of Fukui, Fukui 910-1193, Ja- \\ pan, \\ ${ }^{3}$ Laboratory of Aquatic Natural Products Chemistry, Graduate School of Agricultural and Life Sciences, \\ The University of Tokyo, Bunkyo-ku, Tokyo, 113-8657, Japan
}

KEYWORDS. diffusion limitation, electric field, nano-pore, planar lipid bilayers, single-channel current recordings, waterchain.

\begin{abstract}
A hydrogen-bonded water-chain in a nano-tube is highly proton conductive, and examining the proton flux under electric fields is crucial to understanding the one-dimensional Grotthuss conduction. Here, we exploited a nano-tube-forming natural product, the peptide polytheonamide B (pTB), to examine proton conduction mechanisms at a single-molecule level. The pTB nanotube has a length of $\sim 40 \AA$ that spans the membrane and a uniform inner diameter of $4 \AA$ that holds a single-file water-chain. Singlechannel proton currents were measured using planar lipid bilayers in various proton concentrations and membrane potentials $( \pm 400$ $\mathrm{mV})$. We found, surprisingly, that the current-voltage curves were asymmetric with symmetric proton concentrations in both solutions across the membrane (rectification). The proton flux from the C-terminal to the N-terminal end was 1.6 times higher than the opposite. At lower proton concentrations, the degree of rectification was attenuated, but by adding a pH-buffer (dichloroacetate) that supplies protons near the entrance, the rectification emerged. These results indicate that the permeation processes inside the pore generate the rectification, which is masked at low concentrations by the diffusion-limited access of protons to the pore entrance. The permeation processes were characterized by a discrete-state Markov model, in which hops of a proton followed by water-chain turnovers were implemented. The optimized model revealed that the water-chain turnover exhibited unusual voltage dependence, and the distinct voltage-dependencies of the forward and backward transition rates yielded the rectification. The pTB nano-tube serves as a rectified proton conductor, and the design principles can be exploited for proton-conducting materials.
\end{abstract}

\section{Introduction}

Proton transfer across membranes is a critical issue for both biological and material sciences, and the mechanism underlying the transfer has been studied extensively. ${ }^{1}$ Across biomembranes, several types of membrane proteins (proton pumps, ${ }^{2-4}$ proton transporters and proton channels) participate in proton transfer via distinct molecular mechanisms, thus contributing to cellular functions. Compared to transporters and pumps, channels allow the most efficient permeation because channels provide a water-filled pore through which protons can permeate passively down the electrochemical potential gradient. Several types of proton channels exist in biomembranes for various physiological and pathological functions., ${ }^{5,6}$ For instance, the voltage-gated proton channel protein is expressed in various types of cells and exhibits many physiological functions. ${ }^{5,7-9}$ The mechanism underlying proton permeation has been studied extensively. ${ }^{10-12}$ On the other hand, the M2 protein from the in- fluenza virus forms a proton-conductive channel in the membrane of infected cells, which has also been extensively studied. ${ }^{13-16}$ In material science, proton conductors are mostly solid materials, ${ }^{17}$ and efficient proton transfer is a prerequisite, especially for fuel cells. ${ }^{18}$ Thin membranes of polymer Nafion are a typical proton conductor, ${ }^{19}$ and further development remains necessary.

There is a common unsolved issue in the biological and material sciences, i.e., the mechanism underlying proton permeation. To address these interdisciplinary issues, studying proton channels of biological origin is informative regarding the underlying principles of proton permeation. Among the biological resources exhibiting proton permeation in the membrane, here, we exploit a channel peptide that has much simpler structural features than channel proteins while retaining fundamental functions. The proton permeation process has been studied extensively using the peptide channel gramicidin $\mathrm{A}$, and various 
mechanistic insights have been obtained. ${ }^{20-23}$ Here, we introduce a peptide channel called polytheonamide B (pTB) ${ }^{24}$ because it serves as an ideal "test tube" for studying proton conduction.

pTB is a cytotoxic peptide from a marine sponge, Theonella swinhoei. Its strong cytotoxicity originates from its channelforming activity in target cell membranes. ${ }^{25,26}$ The pTB peptide consists of 48 amino-acid residues of alternate L- and D-chirals with several unusual amino-acid side-chains (Fig. 1 legend). It forms a right-handed $\beta^{6.3}$-helix ${ }^{27,28}$ in which the backbone of the peptide forms a non-interrupted hydrogen-bonded network in the pattern of a parallel $\beta$-sheet (Fig. 1A). The pTB channel has a long (approximately $40 \AA$ ) cylindrical pore that spans the membrane with a single molecule. The inner diameter is $4 \AA$ throughout the pore, and the embedded single-file long waterwire interacts somewhat with the pore wall. The pTB channel also has unusual structural features. ${ }^{29}$ The side chains are located outside of the backbone nanotube (Fig. 1A). There are several amide side chains that are mostly modified, such as methylasparagine (Asm) and methylglutamine (Mgn) (see Fig. 1 legend). They are distributed periodically (one in six residues) on the peptide sequence and are thus aligned along one side of the $\beta^{6.3}$-helix, where they form a hydrogen-bonded strand outside the pore (Fig. 1B). ${ }^{26}$ One long and two short strands exist, which reinforce the stability of the long helical structure. In the $\beta^{6.3}$-helix, the backbone amides are oriented such that their amide dipoles point along the axis in alternate directions; thus, the sum of the dipole moment of the backbone amides is null. ${ }^{28} \mathrm{In}$ contrast, the side-chain amide dipoles generate a net dipole moment along the hydrogen-bonded strands that runs nearly parallel to the channel axis. These unprecedented structural and electrostatic features of the pTB channel provide a specific environment for proton permeation. In this test tube, proton transfer along the water chain was subjected to the influence of the side chain amide dipoles and the membrane electric field (Fig. 1C).

In the Grotthuss mechanism of bulk water, no single proton must move through distances much greater than the average separation between water molecules; only the bonds between hydrogens and oxygens must be rearranged (proton hop). ${ }^{30}$ This mechanism is shared by proton permeation through a hydrogenbonded water wire (single-file water chain) ${ }^{31}$ in a nanotube. In one-dimensional Grotthuss conduction, having participated in the proton hop, all the water molecules in the single-file pore were reversed in their orientation. ${ }^{32}$ Thus, the return of these water orientations is the final step that completes the cyclic permeation process for the single proton transfer across the membrane, by which a coming proton can be accepted for the next permeation cycle.

In this study, proton conduction through the pTB channel was examined using single-channel current recordings in planar lipid bilayers. We found, surprisingly, that the current-voltage curves exhibited weak rectification, even in symmetric $\mathrm{pH}$ solutions, which has never been previously reported for proton conduction through channels. To address the underlying mechanism of the rectification, single-channel currents under various $\mathrm{pH}$ conditions over a wide range of membrane potentials were recorded, and the data were analyzed using a permeation model. The results indicated for the first time that asymmetric water turnovers contribute to the asymmetric proton permeation. According to this underlying mechanism of proton conduction, the pTB channel can serve as a template for designing intelligent cytotoxic drugs or proton conductors in fuel cells.
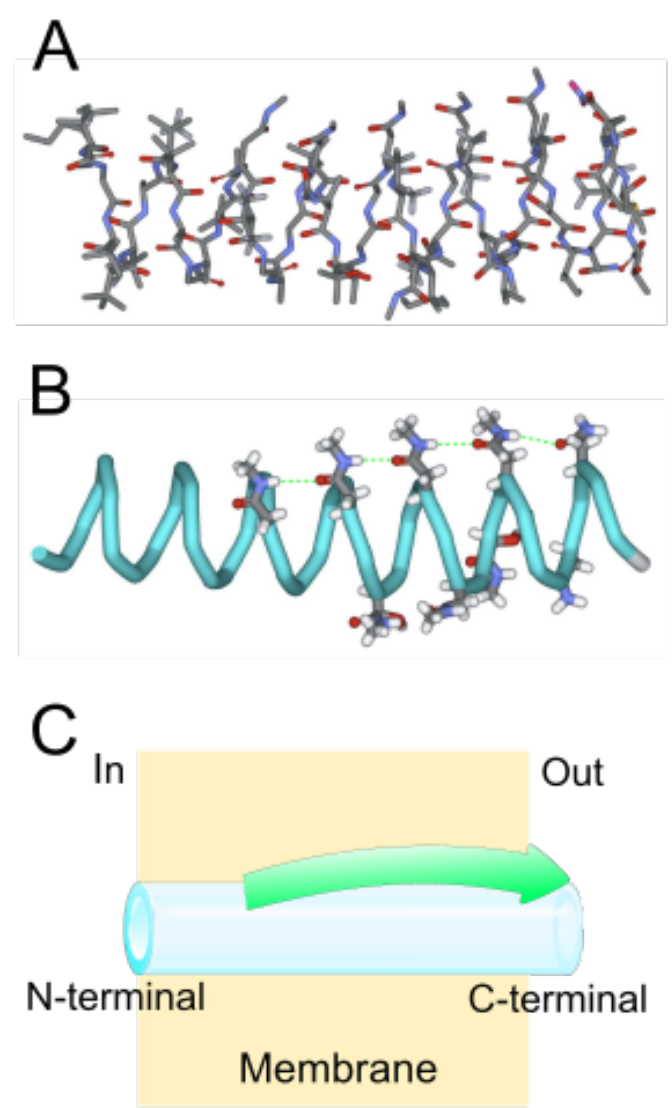

Figure 1. The structure of the pTB channel. A. The structure of the pTB channel. The amino acid sequence of pTB is as follows: Gly-L-Mil-Gly-L-Tle-D-Tle-L-Tle-D-Ala-L-TleD-Tle-L-Ala-Gly-L-Ala-D-Tle-L-Ala-D-Asm-L-HvaGly-L-Ala-Gly-L-Tle-D-Asm-L-Mgn-D-Hva-L-Ala-GlyGly-D-Asm-L-Ile-D-Ham-L-Tle-D-Hva-Gly-D-Asm-LIle-D-Asm-L-Val-D-Ham-L-Ala-D-Asm-L-Val-D-Ser-LVal-D-Asn-L-Mme-D-Asn-L-Gln-D-aTh-L-Thr (Mil: $\beta$ methylisoleucine, Tle: tert-leucine, Asm: $\gamma$-N-methylasparagine, Hva: $\beta$-hydroxyvaline, Mgn: $\beta$-methylglutamine, Ham: $\gamma$-N-methyl-threo- $\beta$-hydroxyasparagine, $\quad$ Mme: $\beta, \beta$-dimethylmethionine sulfoxide, aTh: allo-threonine). The N-terminus is shown at the left. PDB code: 2RQO. B. Schematic representation of the pTB channel. The inner diameter is $4 \AA$. Outside the $\beta^{6.3}$-helix, hydrogen-bonded strands formed by dipolar side-chains run nearly parallel to the pore axis. C. Scheme of the pTB test tube embedded in a membrane. When the pTB tube is added to the extracellular space of the targeted cells (out), it is inserted into the relevant cell membrane with its N-terminal end as the lead. The arrow alongside the tube shows the dipole of a long hydrogen-bonded side-chain strand with its arrow head toward the positive electrical end.

\section{Results}

\section{Rectified proton conduction in symmetric $\mathrm{pH}$ solu- tions}

The single-channel current was measured in planar lipid bilayers formed with diphytanoylphosphatidylcholine (Fig. 2A, Methods). Both compartments contain the same proton concentration (symmetric $\mathrm{pH}$ solutions), and pTB was added in the cis 
compartment. The pTB peptide was spontaneously incorporated into the membrane with its hydrophobic N-terminal end as the lead, and it was oriented such that the N-terminal end of the pTB channel faces the trans compartment (Fig. 1C). ${ }^{26}$ The membrane potential was defined as the potential of the trans side relative to the cis side (Fig. 2A). The current flowing from the cis to the trans side or the $\mathrm{C}$-terminal to $\mathrm{N}$-terminal direction through the channel was conventionally defined as the "inward" current with a negative sign.

The single-channel current traces for the symmetric $\mathrm{HCl}$ solutions ( $\mathrm{pH}$ 1.5/1.5: cis side $\mathrm{pH} /$ trans side $\mathrm{pH}$ ) demonstrate that the pTB channel exhibits fast gating (Fig. 2B). The open dwell time was long at positive potentials and very brief at negative potentials. The single-channel current was recorded at a high temporal resolution (a cut-off frequency of $5 \mathrm{kHz}$ was used).
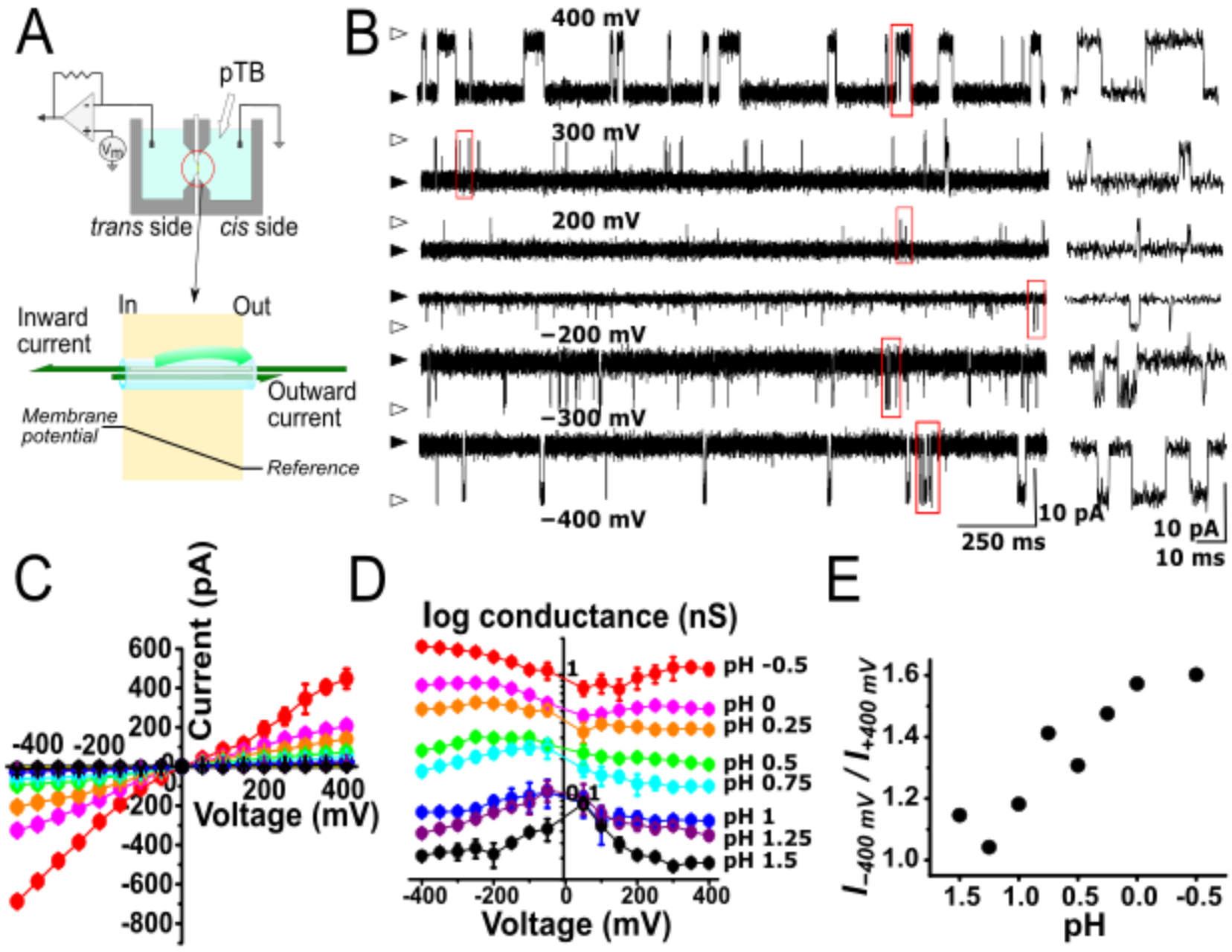

Figure 2. Single-channel proton current of the pTB channel in the planar lipid bilayer. A. The configuration of the experimental setup for the single channel current recordings of the planar lipid bilayers. The pTB peptide was added to the cis compartment, and the channel was spontaneously inserted into the membrane and oriented with the $\mathrm{N}$-terminal end facing the trans side. The membrane potential was defined for the trans side relative to the cis side, and this definition mimics the cell membrane potential of a toxin-targeted cell. The current flowing from the $\mathrm{C}$-terminal to $\mathrm{N}$-terminal was defined as the inward current. B. Representative single-channel current traces at various membrane potentials ( $\mathrm{pH}$ 1.5). The channel opens frequently and stays open for long periods above $300 \mathrm{mV}$. At positive potentials the channel opens with a higher open probability than at other potentials. Infrequent and short openings were observed at negative potentials. The filled arrow heads represent the closed level, and the open heads indicate the open level. The right panel shows traces with an expanded time scale for the red boxed regions. The currents were recorded through the low-pass filter with a cut-off frequency of $5 \mathrm{kHz}$ (the sampling frequency was $25 \mathrm{kHz}$ ), but for the display of the traces, the data were digitally filtered (Butterworth filter) at a $1 \mathrm{kHz}$ cut-off frequency. C. The single-channel current-voltage $(i-V)$ curves at symmetric $\mathrm{pH}$ from 1.5 to -0.5 . The curves show a slight inward rectification at high proton concentrations. Error bars are shown for all points, and many are not visible because of the small size within the symbols. D. The log[conductance]-voltage curves. At high concentrations, the maximum conductance was attained at negative potentials, giving an inward rectification. However, at low concentrations, the conductance was nearly equal at extreme positive and negative potentials, and the maximal conductance was observed at near zero potential. This behavior is a result of the access resistance, which becomes negligible when the current amplitude is small (see the section on diffusion-limited access toward the pore entrance). E. The degree of rectification. The ratio of single-channel current amplitudes at $-400 \mathrm{mV}$ to the amplitudes at $+400 \mathrm{mV}\left(I_{-}-400 \mathrm{mV} / I+400\right.$ $\mathrm{mV}$ ) is plotted as a function of $\mathrm{pH}$. 
Briefly opened channels at negative potentials are shown with an expanded time scale (right panel), exhibiting the rectangular shape of the single-channel current, from which the singlechannel current amplitudes were measured (see Methods for details). The membrane was stable even under severely acidic conditions and extreme membrane potentials (see SI 1, Fig. S1).

The single-channel current-voltage $(I-V)$ curves of the pTB channel were obtained for a wide range of membrane potentials from $-400 \mathrm{mV}$ to $+400 \mathrm{mV}$ (Fig. $2 \mathrm{C}$ ). At $\mathrm{pH}-0.5$, the singlechannel current amplitude reached approximately $400 \mathrm{pA}$ at $+400 \mathrm{mV}$, which is more than ten times greater than any other monovalent cation conductance through the pTB channel $\left(\mathrm{i}_{\mathrm{H}}{ }^{+}\right.$ $\left.>40 \times i_{\mathrm{Cs}}+\right)$, ${ }^{26}$ indicating that the proton permeates via the Grotthuss mechanism. The $I-V$ curves were also obtained for asymmetric $\mathrm{pH}$ conditions (SI 2, Fig. S2), and the reversal potentials for different $\mathrm{pH}$ conditions indicate that the pTB channel is purely proton selective over $\mathrm{Cl}^{-}$.

For the symmetric $\mathrm{pH}$ conditions at $\mathrm{pH}-0.5 /-0.5$, surprisingly, the shape of the $I-V$ curve was inward-rectified (inward current $>$ outward current; Fig. 2C). Rectified permeation of a proton has never been previously observed for ion channels. Given the orientation of the membrane-inserted pTB channel, the proton flux from the $\mathrm{C}$-terminal end to the $\mathrm{N}$-terminal end (the inward current) is greater than in the opposite direction (Fig. 2A). The degree of rectification was expressed as the absolute ratio of the current amplitudes at $-400 \mathrm{mV}$ and $+400 \mathrm{mV}\left(I_{-400 \mathrm{mV}} / I_{+400 \mathrm{mV}}\right)$, and the value was approximately 1.6 (Fig. 2E).

To observe the rectification more clearly under different $\mathrm{pH}$ conditions, the $\log [$ conductance]-voltage $(\log [g]-V)$ curves are shown (Fig. 2D). The curve patterns differ substantially for different proton concentrations. The conductance exhibited maximal values at extreme negative potentials when the proton concentration was high (at $\mathrm{pH}-0.5$; rectification). At low concentrations, however, the maximal conductance was near the zero potential, and the conductance at extreme positive and negative potentials exhibited similar values, indicating no rectification. In fact, at $\mathrm{pH} 1.5 / 1.5$, the $I-V$ curve is apparently linear (Fig. 2C and $4 \mathrm{~B})$. The degree of rectification $\left(I_{-400 \mathrm{mV}} / I_{+400 \mathrm{mV}}\right)$ was plotted as a function of proton concentration (Fig. 2E), indicating that the rectification was gradually attenuated at low proton concentrations. Generally, rectification has been attributed to the asymmetric shape of the potential profile for permeating ions inside the pore or the distinct rate constants for the forward and backward transitions. ${ }^{33}$ Thus, the variable degree of the rectification at different proton concentration is unexpected, which is a challenging issue to solve for the underlying mechanism.
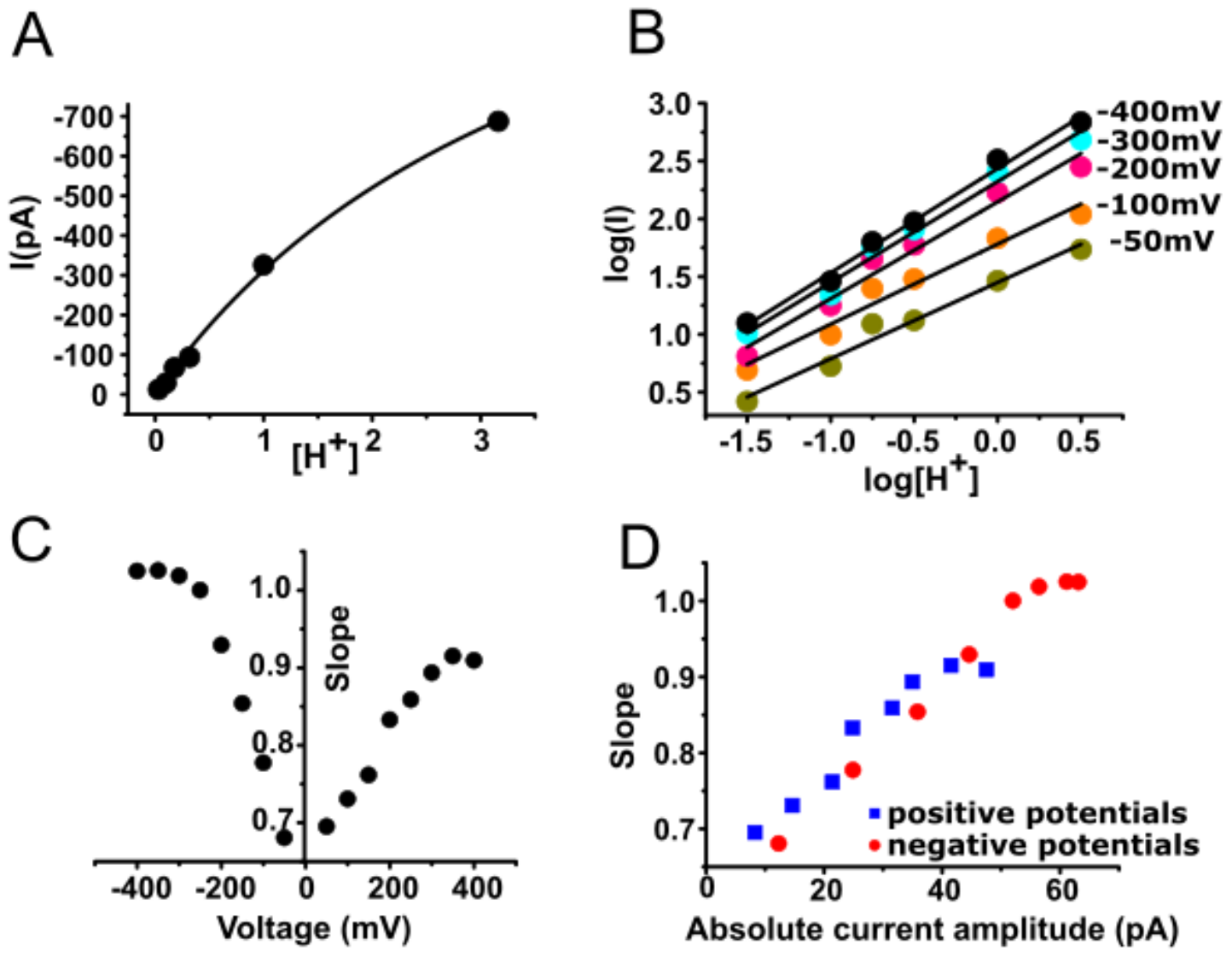

Figure 3. Concentration dependencies of the single-channel proton current through the pTB channel. A. The proton concentration dependency of the current at $-400 \mathrm{mV}$. The proton concentration is linearly scaled on the abscissa. The data were fitted with the MichaelisMenten curve. B. The log-log plot or pH-log[current] plot of the pTB channel at various membrane potentials. The linear fit was performed at the mid-pH range, and the slope was obtained. C. The slope-voltage curve. The slope of the concentration dependency as a function of the membrane potential. The slope obtained from the log-log plot at different membrane potential was used. D. The absolute current amplitude-dependency of the slope. The slope value was plotted as a function of the absolute value of the current amplitude at $\mathrm{pH}$ 0.5. The blue symbols represents the s value at positive potentials, and the red ones for the negative potentials. 


\section{Diffusion-limited access towards the pore entrance}

To examine the concentration-dependent rectification, the single-channel current amplitude at different proton concentrations was investigated (Fig. 3A). As a convention, hereafter, the proton or $\mathrm{H}_{3} \mathrm{O}^{+}$concentration in aqueous solution is expressed as $\left[\mathrm{H}^{+}\right]$. The curve shows the saturating tendency at $3 \mathrm{M}\left[\mathrm{H}^{+}\right]$, suggesting a one-ion pore permeation characteristic that is similar to the one for monovalent cations through the pTB channel. ${ }^{33}$ Fig. 3B shows the transformation of Fig. 3A as the loglog plot or log $(I)-\mathrm{pH}$ plot, and an almost linear relationship is apparent. ${ }^{34}$ From the log-log plot, the slope value (s) was calculated. The $s$ value is an index that indicates the bimolecular association between the proton and the channel. When the subsequent permeation steps inside the pore are more efficient than the proton access towards the pore (see SI 3), the current amplitudes should vary linearly with the proton concentration that has an $s$ value that approaches one, i.e., the diffusion-limited process. ${ }^{36}$ Fig. $3 \mathrm{C}$ shows the $s$ value as a function of the membrane potential. As the membrane potential becomes negative, the $s$ value approaches one, but not at positive potentials, indicating that the diffusion-limited process predominates at extreme negative potentials. One may consider that the access is more limited to the pore opening on the cis side because of its small size. From the structure of the pTB channel, however, the size of the pore openings of both sides are nearly identical (Fig. 1). Then, why is the access limited for the inward current even if the inward current is larger than the outward current?

The $s$ value was plotted as a function of the absolute value of the single-channel current amplitude (s- $|I|$ curve; Fig. 3D). The data for the negative (red symbols) and positive (blue symbols) potentials exhibited a monotonically incremental function and nearly overlapped. This finding suggests that the $s$ value depends on the current amplitude rather than the direction of the current flow. In proton permeation, the throughput rate inside the pore is so high relative to the values for alkali cations that the proton supply from the bulk becomes limited The convergence of the proton flux towards the small entrance of the pore generates additional resistance called the access resistance (Fig. 4A, upper panel; SI 3), and this process becomes the rate-determining step. The access resistance ( $1 /$ [the diffusion-limited rate constant]) increases as the net flux increases, which is formulated as Eq. 2 (Methods). ${ }^{35}$ Thus, the overlapping of the $s$ values for the inward and outward currents suggests that permeation is governed by similar access processes for both sides.

To quantify the access resistance, the diffusion limitation towards the small opening was evaluated using the capture radius ( $r_{\mathrm{c}}$; Eq. 1). From the saturating current amplitude at $-400 \mathrm{mV}$ in $\mathrm{pH}-0.5$ solution, and given the diffusion coefficient of $\mathrm{H}^{+}$ $\left(9.3 \times 10^{-5} \mathrm{~cm}^{2} / \mathrm{s}\right), r_{\mathrm{c}}$ was evaluated to be $0.70 \AA$, which is very close to the value for the gramicidin A channel that has a similar $\beta^{6.3}$-helix pore structure. ${ }^{36}$

\section{Elimination of the access resistance unmasked in- tra-pore rectification}

To circumvent the diffusion limitation and examine the intrapore process straightforwardly, the method developed by Decker and Levitt was applied. ${ }^{20}$ In their experiment, a high concentration of protonatable/deprotonatable buffer substance was added to both sides of the solutions. When the $\mathrm{pH}$ of the solution is identical to the $\mathrm{pK}$ value of the buffer, half of the buffer is protonated, and the other half is deprotonated. The buffer substances in the vicinity of the pore entrance provide protons to the pore, and when deprotonated, protons are continually supplied from the nearby protonated buffers in a bucketbrigade fashion (facilitated diffusion ${ }^{37}$, Fig. 4A, lower panel). Protons accumulate at the pore exit, and are scavenged by the buffer. These processes provide an alternate proton supply route that is parallel to the diffusion-limited process (see SI 4). Thus, the measured current in the presence of the buffer more clearly reflects the intra-pore permeation process by circumventing the diffusion limited path.
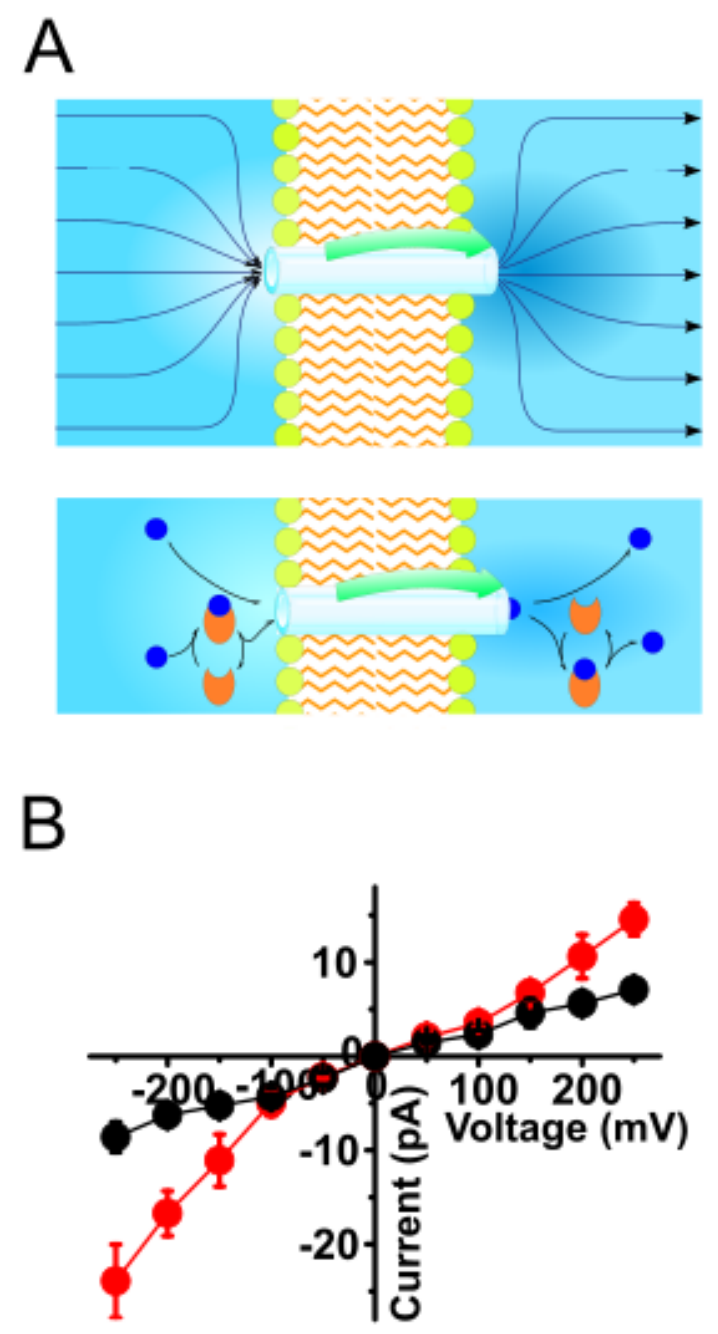

Figure 4. Access resistance and the buffer effect. A. Scheme for the buffer action at the pore entrance and exit. In the absence of the buffer (upper panel), the proton flux converges towards the small opening of the pore entrance. Once it reaches the capture radius, which is smaller than the geometric radius, the proton does not return to the bulk solution. Efficient permeation inside the pore leads to depletion of proton at the entrance: the diffusion limitation. In the presence of a highconcentration of buffer (dichloroacetic acid; orange; lower panel), the protons (blue) are provided from nearby buffer substances at the entrance in a bucket-brigade fashion. At the exit, the buffer scavenges accumulated protons. The color (blue) gradient of the aqueous solution represents the local proton concentration. B. The single-channel current-voltage curve in the absence (black) and presence (red) of the buffer. The concentration of dichloroacetic acid on both sides of the membrane was $500 \mathrm{mM}$ at $\mathrm{pH} 1.5$. 
Here, we used dichloroacetate as the buffer (pKa value $=1.48$; $500 \mathrm{mM}$ ) on both sides of the solutions at $\mathrm{pH} 1.5$, and the single-channel current was recorded. Dichloroacetate destabilizes the membrane and the membrane potential could not be applied beyond $\pm 250 \mathrm{mV}$. The single-channel $I-V$ curve is shown in Fig. 4 , which is superimposed on the $I-V$ curve in the absence of the buffer at $\mathrm{pH}$ 1.5. The conductance was more than twice as large as in the control condition, and inward rectification appeared. Thus, the results indicate that when protons are sufficiently supplied to the pore, the permeation feature reflects the process inside the pore, indicating that the rectification originates from conduction processes inside the pore.

\section{Proton-hop and water-turnover model under the in- fluence of the membrane electric field}

The current rectification is now considered to be a manifestation of the one-dimensional Grotthuss conduction inside the pore, which is characterized by the proton hopping steps across the water-wire, followed by water turnover. ${ }^{38,39}$ This theoretical framework has been used exclusively for understanding proton permeation through channels, including in the gramicidin A channel, ${ }^{38-40}$ even though they are not rectified. To relate the present experimental results to the theory, underlying elementary steps, including the diffusion-limited access process, were implemented, and these distinct processes of proton permeation were integrated into the model to account for the rectification. Before addressing the specific model, the general framework of the model is explained. We applied a minimal model with a small number of states and transitions between them (a discretestate Markov model; DSMM), ${ }^{42,43}$ which is similar to the lumped state model proposed by Schumaker et al. for the hopand-turn mechanism. ${ }^{41}$ The model is cyclic in its nature, and cyclic transitions among states yield proton currents. Accord-

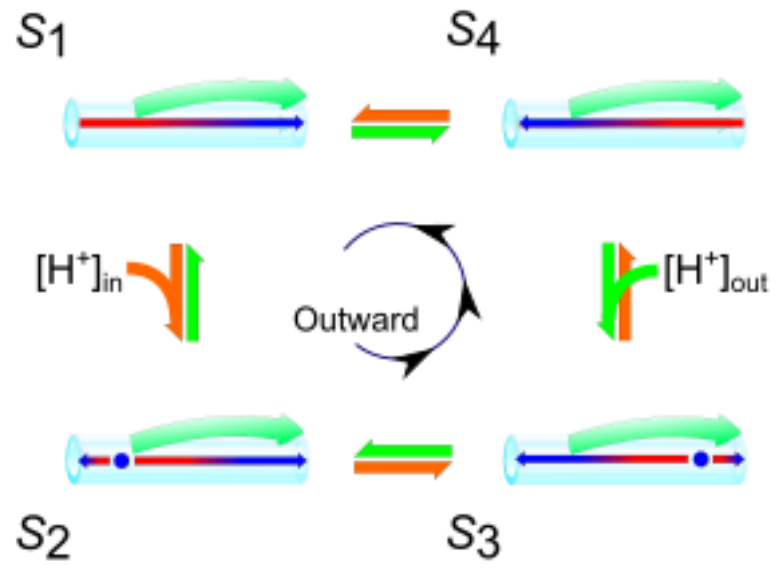

Figure 5. The four-state proton permeation models for the pTB channel. The permeation process is expressed as the transitions among four states. The dark blue sphere indicates a proton. The colored arrows in the pore indicate the directions of lumped water dipoles (blue for positive and red for negative ends). The arrows between states represent transition directions (orange for outward and green for inward). $\left[\mathrm{H}^{+}\right]$in represents the $\mathrm{H}^{+}$ concentration in the intracellular side and $\left[\mathrm{H}^{+}\right]_{\text {out }}$ indicates that in the extracellular side. ingly, the hop-and-turn mechanism is decomposed into the elementary steps, and there should be a critical step for the preferred directional flux that is responsible for the rectification.

The implementation of the theory into the model is readily understood by tracing the permeation process, and we employed the four-state model (Fig. 5): two states are the proton-bearing states inside the pore, which are frequently called excess proton states $\left(S_{2}\right.$ and $\left.S_{3}\right)$, and the other two states are water-filled $\left(S_{1}\right.$ and $S_{4}$ ). Here, the permeation process of, for example, the outward current at a positive potential (the counter-clockwise transitions; Fig. 5) is traced. A proton accesses the entrance of the $\mathrm{N}$-terminal end $\left(S_{1} \rightarrow S_{2}\right)$. To accept an incoming proton, water molecules in the pore are oriented such that the oxygen atoms point towards the entrance. These water molecules are lumped into one group in the $S_{1}$ state (the red and blue arrow), and the arrow head (positive) points right, or toward the C-terminal end (Fig. 5). After the proton enters the pore (blue sphere in the $S_{2}$ state), the water molecules that are upstream of the proton reverse their dipoles (the reversed arrow to the left of the proton), whereas the water molecules downstream of the proton maintain their dipole orientation. Thus, the water chain is separated into two groups: the short upstream chain and the long downstream chain. The excess proton subsequently hops along the water chain, leading to the $S_{3}$ state, in which a small number of water molecules in the downstream of the proton still retain their dipole orientation. After the proton exits the pore towards the bulk solution, all of the water molecules in the pore are reversely oriented (the $S_{4}$ state) compared to the $S_{1}$ state. Subsequently, the water molecules turn their orientation at a time and return to the initial $S_{1}$ state, completing the cyclic reaction. The reversal of the orientation makes the single-file water ready to accept the next proton.

In these processes, the membrane electric field affects all of the transition rates between the states (orange arrows for the forward [or counter-clockwise] transitions and greens for the backward [or clockwise]), and the voltage dependency of each rate constant is expressed by the fractional electric energy transfer factors, $f^{*}$ (Eq. 7a, b in Methods; see SI 5). ${ }^{45,46}$ For the transition from the empty pore to the excess proton state $\left(S_{1} \rightarrow S_{2} ; S_{4}\right.$ $\rightarrow S_{3}$ ), a diffusion-limited proton access step towards the pore entrance was implemented using the conventional formula (Eq. 2 - 4 in the Methods section), which takes into account the capture radius and the diffusion-limited ion permeability, $p_{\mathrm{a} .}{ }^{35} \mathrm{Mul}-$ tiple proton occupancy in the pore was not considered ${ }^{44}$ because the concentration-dependent current amplitude exhibited saturation behavior at a high proton concentration (Fig. 3A). The net proton flux was calculated using Eq. 8 (Methods).

\section{Model analysis revealed an asymmetric water turn- over process}

The four-state model served to optimize the rate constants for all of the single-channel current data, including the $I-V$ data for the symmetric and asymmetric $\mathrm{pH}$ conditions. The optimization using the Nelder-Mead algorithm (Methods) was performed by systematically varying the initial parameter sets. ${ }^{47}$ We also examined a three-state model and other types of the four-state model (SI 6, Fig. S3). The optimized parameters produced a good $I-V$ curve fit at different $\mathrm{pH}$ values (SI 2, Fig. S2). The goodness-of-fit was substantially increased for the four-state model in Fig. 5 relative to the three-state model and the different four-state model. Thus, we selected the four-state model to represent proton permeation through the PTB channel. 

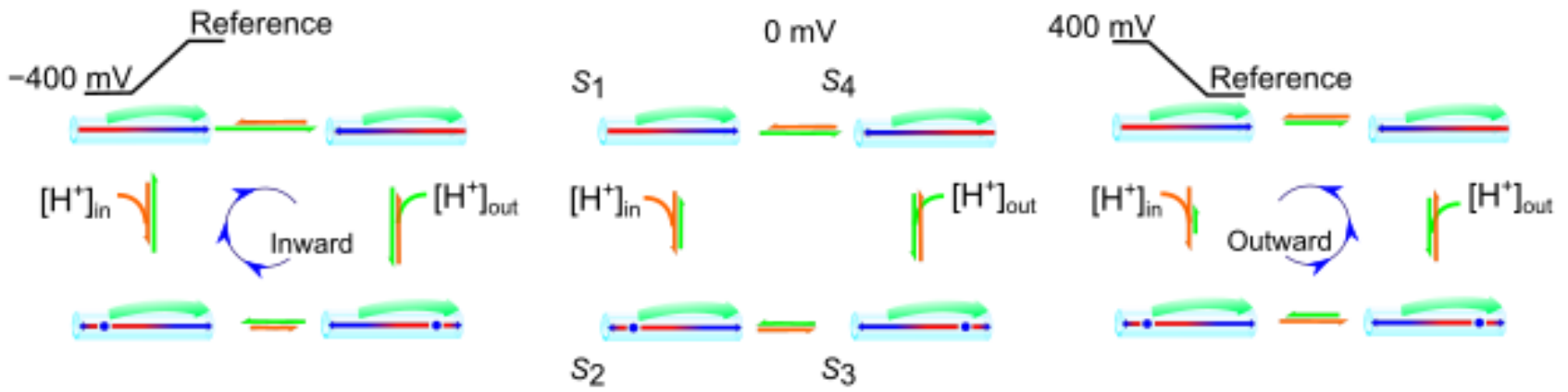

Fig. 6. The voltage dependence of the rate constants for the four-state model. The amplitude of the rates is represented by the arrow length at $-400,0$ and $+400 \mathrm{mV}$.

Table 1 . The rate constants for the four-state model.

\begin{tabular}{|c|c|c|}
\hline & $k^{0}\left(\times 10^{6} / \mathrm{ms}\right)$ & $f^{*}$ \\
\hline$k_{12}$ & 4.65 & 0.0082 \\
\hline$k_{23}$ & 1.42 & 0.147 \\
\hline$k_{34}$ & 24.5 & 0.0001 \\
\hline$k_{41}$ & 20.5 & -0.043 \\
\hline$k_{21}$ & 0.475 & 0.385 \\
\hline$k_{32}$ & 1.55 & 0.081 \\
\hline$k_{43}$ & 11.4 & 0.128 \\
\hline$k_{14}$ & 398.0 & 0.294 \\
\hline
\end{tabular}

The rate constants are expresses as Eq. 7a and b. For $k_{12}$ and $k_{41}$, they are modified by the net flux (see Eq. 2).

During the optimization process, the $f_{41} *$ value for the water turnover transition of $S_{4} \rightarrow S_{1}$ frequently became negative. We initially ignored this negative parameter because previous model studies for varieties of channels have never reported negative $f^{*}$ values. ${ }^{46}$ Repeated optimizations, however, gave the negative value exclusively for $f_{41}$, and the goodness-of-fit improved substantially.

The transition rates at different membrane potentials are schematically shown in Fig. 6 in which the orange and green arrows represent the forward and backward rate constants, respectively, with their lengths representing the amplitudes of the voltagedependent transition rates. The parameters for the rate constants (Eq. 7a, b) are shown in Table 1 . The sum of the $f^{*}$ values for the inward direction $\left(k_{21}+k_{32}+k_{43}+k_{14}=0.89\right)$ overwhelms that of the opposite $\left(k_{12}+k_{23}+k_{34}+k_{41}=0.11\right)$. All of the backward (or inward) rates increased at the negative potentials (longer arrow length), and the opposite was true for the forward (or outward) rates, except for one, $k_{41}$, which increased at negative potentials because of the negative $f_{41}$ * The underlying mechanism that is related to the rectification is discussed below.

\section{Discussion}

The pTB channel is a chemically robust peptide, maintaining its channel function even at $\mathrm{pH}-0.5$, and our stable planar lipid bilayers allowed single-channel current measurements in the voltage range of $\pm 400 \mathrm{mV}$. The pTB channel serves an ideal test tube for examining the behavior of the proton transfer through a hydrogen-bonded single-file water chain under the influence of the membrane electric field. We found for the first time that proton permeation through the pTB channel manifests rectified conduction. Even at low proton concentrations in which diffusion-limited access resistance predominates, the rectification emerged after the $\mathrm{pH}$-buffer dichloroacetate was added to the solutions in high concentration, which circumvented the access diffusion. These results unequivocally indicate that the rectification originates from the intra-pore processes. In fact, the $\mathrm{pH}$ dependent changes in the rectification (Fig. 2E) are a result of different contributions from the access diffusion and the intrapore processes. Similarly, the distinct patterns of the conductance-voltage curves (Fig. 2D) can be readily explained: The maximum value at extreme negative potentials in high proton concentrations reflects the rectifying property inside the pore, whereas at low proton concentrations the limited proton supply renders the flux diffusion-limited at both extreme positive and negative potentials. However, at around zero membrane potential, the low net flux makes the contribution from the access resistance negligible and results in a relatively high conductance.

To understand the origin of the rectification for one-dimensional Grotthuss conduction, the cyclic four-state model links the systematically performed experimental data with the theory. The model that was fit to the data revealed that, among the models, the four-state model was the best-fit model. Although the model is coarse grained and further studies on atomistic levels are required in future works, the simple model does not lose the generality of the Grotthuss mechanism. Additionally, it allowed the extraction of some essential features in the proton permeation process. We pose here a general question regarding the rectification. The rectification is not simply a preferred flux of one direction over the other, but rather the flux of one-direction is augmented much more than the opposite as the membrane potential is applied. Thus, the rectification originates from the voltage dependency of the rates, which is expressed as $f^{*}$. For one-dimensional Grotthuss conduction, the voltage dependency was not fully discussed in earlier studies, and we first examine the processes of the one-dimensional Grotthuss conduction on the four-state model with respect to the electrostatics. Then, the results of the model optimization will be interpreted in relation to the channel and water structure.

The cyclic path in the model is separated into two partial paths: the transition from $S_{1}$ to $S_{4}\left(S_{1} \rightarrow S_{2} \rightarrow S_{3} \rightarrow S_{4}\right)$ represents the proton hop (the hop path), whereas the transition from $S_{4}$ to $S_{1}$ $\left(S_{4} \rightarrow S_{1}\right.$ ) represents the water turnover (the turnover path). In the hop path, the sum of the $f$ values along the path needs not to be one, because it does not simply represent the net transfer of 
one proton charge across the pore, but the contribution of dipole turnover of all of the water molecules in the pore is involved. When we observe the distinct transition of $S_{2} \rightarrow S_{3}$, a proton moves down the electric field, and this hop through the water molecules causes the relevant water molecules to reverse their dipole orientation. These concomitant hop and turn events contribute oppositely to the electrostatic energy level of the states and their transitions. Generally, the electric contribution of the proton charge transfer (charge-electric field) to the free energy is greater than that of the dipole turnover (dipole-electric field), and the $f^{*}$ value should be positive. On the other hand, in the turnover path, the dipole return of the water in the pore is simply governed by the electrostatics of the water dipole, and the dipole of the water chain interacts with the electric field and with the dipoles of the channel. Up to this point, the above considerations have been generally applicable to the one-dimensional Grotthuss conduction, even though the four-state model was used as a framework.

Now, we specifically discuss the optimized four-state model. The model fitting revealed that the sum of the $f^{*}$ values for the inward direction (0.89) overwhelmed that of the outward direction (0.11), and this is the origin of the inward rectification. More specifically, our unexpected finding in this study is that the forward rate constant $\left(S_{4} \rightarrow S_{1}\right)$ is characterized by an unprecedented negative $f_{41}$ * value, and the difference between $f_{41}$ * and $f_{14} *$ values is high. To understand the underlying mechanism of this unusual voltage dependency, the four-state model was redrawn (Fig. 7) with plausible configurations for the proton and water for each state based on thermodynamic and electrostatic considerations and the structural features of the pTB pore. The major sources of the free energy of the states include the following: 1) the dipole-dipole interaction between the sidechain and the water, 2) the dipole-electric field interaction between the water and the membrane electric field, and 3) the proton-side-chain dipole and proton-electric field. Compared to the water turnover that occurs every time a proton permeates across the pore, the orientation of the side chains should be fixed because the motion of the dipolar side chains is restricted relative to the water in the pore. The side-chain dipole was drawn as a fixed dipole arrow (the backbone dipole moment is null on average for the $\beta$-helical structure).

Therefore, we assigned plausible orientations of the water and side chain for each state. In $S_{1}$, the water dipole should be oriented such that oxygen points towards the intracellular (left) side to readily accept an incoming proton, which is reversed in $S_{4}$. In the absence of the applied membrane potential, the free energy level of the states indicates that $S_{4}$ is more stable than $S_{1}$ (SI 7, Fig. S4). This can occur if the side-chain dipole is oriented anti-parallel to the water dipole in $S_{4}$, which is stabilized by the dipole interaction between the reversed water and the side chain. This arrangement of the side-chain dipole is consistent with NMR data ${ }^{27}$ and the results of normal mode analysis, ${ }^{28}$ in which the results were obtained in the absence of a membrane potential. In the process of water turnover $\left(k_{41}\right)$, the negative $f_{41}$ * value indicates that the electric field does not favor the transition process for the water turnover. Thus, it is likely that the water molecules near the dipole side chains are aligned anti-parallel in $S_{4}$ and interact so strongly that a reversal of the relevant water is difficult under the relevant membrane electric field. When negative membrane potentials are applied, the sidechain dipole is destabilized, which facilitates the water turnover because of the attenuated dipole-dipole interaction.

Our hypothetical mechanism for the proton permeation is compared with previously reported results of ion permeation

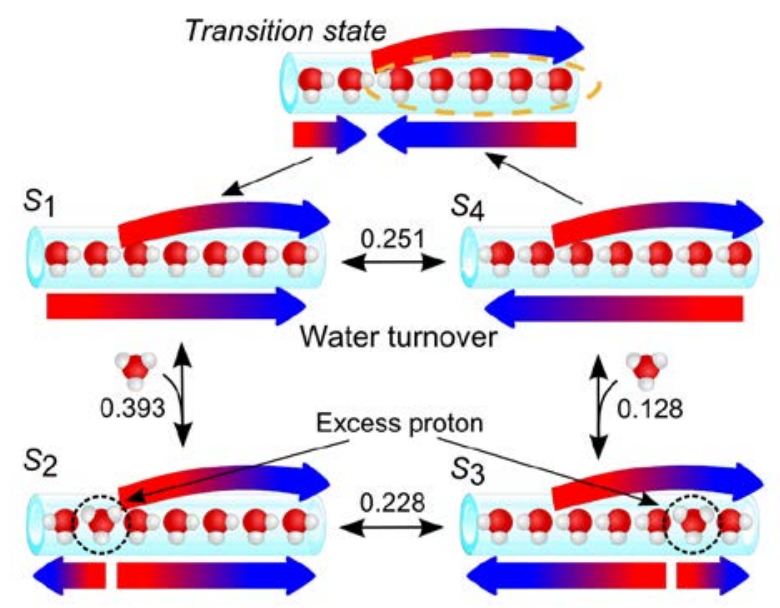

Figure 7. The dipole model for proton permeation. The orientations of water and side-chain dipoles are expressed with the arrows. The number of water molecules in the pore is arbitrary. The number near the transition path indicates the sum of the fractional electric-energy transfer factors $\left(f_{\mathrm{ij}} *+f_{\mathrm{ji}} *\right)$. The transition state (TS) between $S_{4}$ and $S_{1}$ is shown. From $S_{4}$ to $T S$, the transition is unfavorable at positive potentials (negative $f_{41}{ }^{*}$ ). Here, we present a hypothetical arrangement of water dipoles for the transition state. The water molecules in the orange oval are not readily turned over, because these water molecules interact with the side-chain dipoles.

through $\beta^{6.3}$-helical pores. In the gramicidin A channel, ${ }^{48}$ the effects of side-chain dipoles on the permeation were examined, ${ }^{23,49}$ but most of them used homo-dimeric symmetric channels and rectified permeation was not observed. ${ }^{23,34,49}$ On the other hand, we previously showed that introducing a dipolar side chain into one of the dimeric channels (hetero-dimer) produces an asymmetric $I-V$ curve for $\mathrm{Cs}^{+}$permeation. ${ }^{22,49,50}$ In the pTB channel, the $I-V$ curves for $\mathrm{Cs}^{+}$permeation were inwardrectified, and they had a similar degree of rectification as the proton permeation in this study. ${ }^{33}$ Previously, we found that the long pTB channel only hold one alkali cation during permeation (the paradoxical one-ion pore feature), and we hypothesized that strong interactions between water molecules within the channel prevent double ion occupancy. ${ }^{33}$ These results converged to a common process in which the interactions between the side-chain dipole and the water dipole modify the forward and backward rate distinctly regardless of whether the permeating ions are proton or alkali cations. The effect of water turnover has generally been overlooked in the ion permeation process through the pore, and further studies are necessary.

For the single-channel experiment, chemically robust pTB has not permitted modifications to the side chains. Recently, the total synthesis of pTB was successful; ${ }^{29}$ thus, modifications to the pTB nanopore would generate variable permeation characteristics. For example, the degree of rectification could be augmented or even reversed. If the designed channel shows reverse rectification, the "toxin" will become active only when the target cell depolarizes its membrane potential. In material science, a modified pTB-doped membrane is realistic for the fuel cell because of its tough chemical nature. For both basic and applied science, $\beta^{6.3}$-helical pTB serves an ideal test nano-tube for proton conduction. 


\section{Methods}

\section{Polytheonamide B}

High-performance liquid chromatography-purified polytheonamide B (purity > 98\%) ${ }^{24}$ was dissolved in ethanol and stored.

\section{Single-channel current measurements in the pla- nar lipid bilayer}

Single-channel current measurements were performed primarily using the conventional planar lipid bilayer method ${ }^{26}$. Some experiments were performed using the contact bubble bilayer (CBB) method $^{51}$, and the single-channel current traces were indistinguishable. In all of the experiments, AgCl pellet electrodes (A-M systems, WA, USA) were used. For the conventional method, a small amount of diphytanoylphosphatidylcholine (diPhyPC, Avanti Polar Lipids, AL, USA) dissolved in n-decane $(20 \mathrm{mg} / \mathrm{ml}$, nacalai tesque, Kyoto Japan) was painted on the hole (50 - $100 \mu \mathrm{m}$ in diameter) in the Teflon chamber to form the planar lipid bilayer (Fig. 2A), whereas hexadecane was used to dissolve the diPhyPC (20 $\mathrm{mg} / \mathrm{ml}$ ) for the CBB method. The pTB peptide was added to the cis side (reference electrode side) compartment at a final concentration of approximately $10 \mathrm{pM}$, and we waited until channel activities for a few of the pTB channels appeared. Most of the experiments were performed in unbuffered $\mathrm{HCl}$ solutions ( $\mathrm{pH}-0.5$ to 1.5 ). For the measurements in asymmetric solutions, the liquid junction potential was corrected for the membrane potential ${ }^{52}$. For the experiment with buffer, the concentration of dichloroacetic acid (Wako Pure Chemical Industries, Osaka, Japan) was $500 \mathrm{mM}$ at $\mathrm{pH}$ 1.5. A higher concentration of dichloroacetic acid could not be used due to instability of the membrane. The single-channel current amplitude was obtained from the all-point histogram. When the opendwell time was very short, an event-detector program was used (pClamp, Molecular Device, CA, USA), and in the open dwell time-current plot, the events with longer dwell times were used for the single-channel current amplitude.

\section{Capture radius}

The capture radius, $r_{\mathrm{c}}$, is the radius upon which an approaching proton converges and does not return to the bulk once attaining this radius. The $r_{\mathrm{c}}$ was calculated using the following equation. ${ }^{53}$

$$
J_{X}=2 \pi r_{c} D_{X}\left[X^{+}\right] \quad \text { Eq. } 1
$$

where $J_{\mathrm{X}}$ is the cation flux and $D_{\mathrm{X}}$ is the diffusion coefficient of $\mathrm{X}$ cation.

\section{The diffusion-limited rate constants}

The diffusion-limited process was implemented using the calculated capture radius. Given the $r_{\mathrm{c}}$ value, the diffusion-limited process outside the pore was quantitatively evaluated. ${ }^{35}$

The rate of proton association steps that approached from both sides of the bulk solution towards the channel entrance is generally expressed as the product of the proton concentration in the bulk solution and the second-order rate constants $\left(k^{*}\right)$. In the case of diffusion-limited processes, the rate is represented as a function of the proton flux, as follows: ${ }^{35}$

$$
\left[\mathrm{H}^{+}\right] k^{*} \rightarrow\left(\left[\mathrm{H}^{+}\right]-J_{\mathrm{H}} / p_{\mathrm{a}}\right) k^{*} \quad \text { Eq. } 2
$$

where $p_{\mathrm{a}}$ is the diffusion-limited ion access permeability,

$$
p_{\mathrm{a}}=2 \pi r_{\mathrm{c}} D_{\mathrm{H}} \quad \text { Eq. } 3
$$

Given the $r_{\mathrm{c}}$ value of $0.7 \AA$ ("see the section of Diffusion-limited access toward the pore entrance";), $p_{\text {a }}$ was $2.47 \times 10^{6}$ $/(\mathrm{ms} \cdot \mathrm{M})$, as determined from Eq. 3. Eq. 2 features the diffusionlimited process such that the effect is ignored at low net flux, whereas the overall rate is attenuated substantially at high net flux.

\section{The rate constant and the fractional electric energy transfer factor}

The cyclic reaction driven by the electrochemical potential difference is formally expressed as: ${ }^{54}$

$$
\frac{\prod k_{i j}^{+}}{\prod k_{j i}^{-}}=\frac{\left[H^{+}\right]_{o}}{\left[H^{+}\right]_{i}} \operatorname{Exp}[\text { e V } / k T] \quad \text { Eq. } 4
$$

where $k_{\mathrm{ij}}{ }^{+}$and $k_{\mathrm{ji}}{ }^{-}$represent the rate constants for the forward transition (contributing positive ionic currents) and the backward one-way cyclic paths, respectively. The $e$ and $k T$ represent the usual thermodynamic constants. Thus,

$$
\frac{k_{12} k_{23} k_{34} k_{41}}{k_{14} k_{43} k_{32} k_{21}}=\frac{\left[H^{+}\right]_{o}}{\left[H^{+}\right]_{i}} \operatorname{Exp}[e V / k T] \quad \text { Eq. } 5
$$

In each step, a proton is transferred or a water dipole is turned over, and the electrochemical potential energy is dissipated step by step. This dissipating process is expressed as the energy transfer between the states under the influence of the membrane potential, which is expressed by the fractional electric-energy transfer factor, $f_{\mathrm{ij}}$.

$$
\frac{k_{i j}}{k_{j i}}=\frac{k_{i j}^{o}}{k_{j i}^{o}} \operatorname{Exp}\left[f_{i j} \text { e } V / k T\right] \quad \text { Eq. } 6
$$

where $k^{o}$ represents the rate constant at a zero membrane potential. The fractional electric-energy transfer factor has also been called the electrical distance. ${ }^{45}$ For each rate constant for the forward and backward reactions, $f_{\mathrm{ij}}$ is further decomposed into $f_{\mathrm{ij}} *$ (forward) and $f_{\mathrm{ji}} *$ (backward) as follows.

$$
\begin{array}{ll}
k_{i j}=k_{i j}^{o} \operatorname{Exp}\left[f_{i j}^{*} \text { eV } / k T\right] & \text { Eq. 7a } \\
k_{j i}=k_{j i}^{o} \operatorname{Exp}\left[-f_{j i}^{*} \text { eV } / k T\right] & \text { Eq. 7b }
\end{array}
$$

where $f_{j i}=f_{j i}^{*}+f_{j i}^{*}$. These factors can be evaluated as free parameters from the model optimization and they provide clues for the underlying electrostatic processes. For the rate constant of the proton access steps ( $k_{14}$ and $k_{43}$ ), $k^{0}$ in the above equations is replaced by Eq. 2, to include the access resistance that depends on the proton flux. 


\section{The flux equation}

The effect of the access resistance was implemented using the relevant second-order rate constants ( $k_{12} *$ and $k_{43} *$; Eq. 2 ). The flux was solved from a cubic equation for the 4-state model, and the following equation gave a flux value of a real number.

$$
J_{H}=-\frac{1}{3 A}\left(B+\frac{(1-i \sqrt{3})\left(B^{2}-A C\right)}{2^{2 / 3} F}+\frac{(1+i \sqrt{3}) F}{2^{4 / 3}}\right)
$$

Eq. 8

where

$$
\begin{aligned}
& A=-k_{12} k_{43}\left(k_{23}+k_{32}\right) \\
& B=p_{a}\left\{\left(k_{21} k_{32}+k_{14}\left\langle k_{21}+k_{23}+k_{32}\right\rangle\right) k_{43}\right. \\
& -k_{12}\left(k_{34} k_{41}\right. \\
& +k_{32}\left\langle k_{41}+\left\{\left[\mathrm{H}^{+}\right]_{o}-\left[\mathrm{H}^{+}\right]_{i}\right\} k_{43}\right\rangle \\
& +k_{23}\left\langle k_{34}+k_{41}\right. \\
& \left.\left.\left.+\left\{\left[H^{+}\right]_{o}-\left[H^{+}\right]_{i}\right\} k_{43}\right\rangle\right)\right\} \\
& C=p_{a}\left\{k _ { 1 4 } \left(k_{21}\left\langle k_{32}\left(p_{a}+k_{43}\right)+p_{a}\left(k_{34}+\left[H^{+}\right]_{o} k_{43}\right)\right\rangle\right.\right. \\
& +p_{a}\left(k_{23}\left\langle k_{34}+\left[H^{+}\right]_{o} k_{43}\right\rangle\right. \\
& \left.\left.+\left[\mathrm{H}^{+}\right]_{o} k_{32} k_{43}\right)\right) \\
& +k_{12}\left(k _ { 2 3 } \left\langlek_{34}\left\{p_{a}\left[H^{+}\right]_{i}+k_{41}\right\}\right.\right. \\
& \left.+p_{a}\left[H^{+}\right]_{i}\left\{k_{43}\left[H^{+}\right]_{o}+k_{41}\right\}\right\rangle \\
& +p_{a}\left[H^{+}\right]_{i}\left\langle k_{34} k_{41}\right. \\
& \left.\left.+k_{32}\left\{k_{41}+\left[H^{+}\right]_{o} k_{43}\right\}\right\rangle\right) \\
& +p_{a}\left(k_{23} k_{34} k_{41}\right. \\
& \left.\left.+k_{21}\left\langle k_{41}+\left[H^{+}\right]_{o} k_{43}\right\rangle\right)\right\} \\
& D=p_{a}{ }^{2}\left(\left[H^{+}\right]_{o} k_{12} k_{23} k_{34} k_{41}-\left[H^{+}\right]_{i} k_{14} k_{43} k_{32} k_{21}\right) \\
& E=\sqrt{-4\left(B^{2}-3 A C\right)^{3}+\left(2 B^{3}-9 A B C+27 A^{2} D\right)^{2}} \\
& F=\left(-2 B^{3}+9 A B C-27 A^{2} D+E\right)^{1 / 3}
\end{aligned}
$$

\section{Model parameter optimization}

For the four-state model, there are eight rate constants involving the voltage-independent factors and the fractional electric-energy transfer factors, but two of these factors are not free because of the thermodynamic requirement. The 14 free parameters were optimized using the Nedler-Mead algorithm ${ }^{47}$ for the extensive initial parameter sets. Among the models, statistical evaluations were performed to select the valid model by using the Akaike information criterion, in which the maximal likelihood values as well as the number of free parameters are considered for the best-fit model (Eq. S3 in SI 5). ${ }^{55}$

All of the algebraic and numerical calculations were performed using Mathematica ver. 10 (Wolfram Research, Champaign, IL, USA).

\section{Dipole calculation}

The $f$ value is the fraction of the energy expressed in milli-eV, which is translated into the reversal of a dipole moment of 72
Debye, given that the membrane potential was applied over the length of $40 \AA$.

\section{ASSOCIATED CONTENT}

\section{Supporting Information.}

1. Stability of the single-channel current recordings.

2. Single-channel I-V curves in asymmetric $\mathrm{pH}$ solutions.

3. Buffer effect

4. The fractional electric energy transfer factor vs. the electric distance

5. Other permeation models and the optimization

6. Potential profile

This material is available free of charge via the Internet at http://pubs.acs.org.

\section{AUTHOR INFORMATION}

\section{Corresponding Author}

* Shigetoshi Oiki

Department of Molecular Physiology and Biophysics

University of Fukui Faculty of Medical Sciences

23-3 Matsuokashimoaizuki, Eiheiji-cho, Yoshida-gun, Fukui 9101193, Japan

E-mail: oiki-fki@umin.ac.jp

\section{Author Contributions}

All authors discussed data and reviewed the manuscript. All authors have given approval to the final version of the manuscript.

\section{Funding Sources}

This work was supported by JSPS KAKENHI grant number 23370067 (to S.O.).

\section{ACKNOWLEDGMENT}

We thank Dr. Takashi Sumikama for critically reading the manuscript, Ms. Chikako Matsuoka and Eri Shimada for technical assistance, and Ms. Tomoko Matsuki for the artwork.

\section{ABBREVIATIONS}

pTB, polytheonamide $B$; $f$, the fractional electric energy transfer factor; I-V curve, current-voltage curve; $p_{\mathrm{a}}$, the diffusion-limited ion permeability.

\section{REFERENCES}

(1) Wraight, C. A. Biochim. Biophys. Acta - Bioenerg. 2006, 1757, 886-912.

(2) Lanyi, J. K. J. Struct. Biol. 1998, 124, 164-178.

(3) Cotter, K.; Stransky, L.; McGuire, C.; Forgac, M. Trends Biochem. Sci. 2015, 40, 611-622.

(4) Okuno, D.; Iino, R.; Noji, H. J. Biochem. 2011, 149, 655-664.

(5) DeCoursey, T. E. Biochemistry 2015, 54, 2350-3268.

(6) Wang, J.; Qiu, J. X.; Soto, C.; DeGrado, W. F. Curr Opin Struct Biol. 2011, 21, 68-80.

(7) Sasaki, M.; Takagi, M.; Okamura, Y. Science 2006, 312, 589592.

(8) Wu, L.-J. Neuroscientist 2014, 20, 599-609.

(9) Sakai, H.; Li, G.; Hino, Y.; Moriura, Y.; Kawawaki, J.; Sawada, M.; Kuno, M. J Physiol 2013, 23, 5851-5866.

(10) Kuno, M.; Ando, H.; Morihata, H.; Sakai, H.; Mori, H.; Sawada, M.; Oiki, S. J. Gen. Physiol. 2009, 134, 191-205.

(11) Takeshita, K.; Sakata, S.; Yamashita, E.; Fujiwara, Y.; Kawanabe, A.; Kurokawa, T.; Okochi, Y.; Matsuda, M.; Narita, H.; Okamura, Y.; Nakagawa, A. Nat. Struct. Mol. Biol. 2014, 21, 352-357. 
(12) Ramsey, I. S.; Mokrab, Y.; Carvacho, I.; Sands, Z. a; Sansom, M. S. P.; Clapham, D. E. Nat. Struct. Mol. Biol. 2010, 17, 869875.

(13) Schnell, J. R.; Chou, J. J. Nature 2008, 451, 591-595.

(14) Liang, R.; Li, H.; Swanson, J. M. J.; Voth, G. a. Proc. Natl. Acad. Sci. 2014, 111, 9396-9401.

(15) Hong, M.; DeGrado, W. F. Protein Sci. 2012, 21, 1620-1633.

(16) Hong, M.; Fritzsching, Keith J. Williams, J. K. J. Am. Chem. Soc. 2012, 134, 14753-14755.

(17) Karim, M. R.; Hatakeyama, K.; Matsui, T.; Takehira, H.; Taniguchi, T.; Koinuma, M.; Matsumoto, Y.; Akutagawa, T.; Nakamura, T.; Noro, S.; Yamada, T.; Kitagawa, H.; Hayami, S. J. Am. Chem. Soc. 2013, 135, 8097-8100.

(18) Peighambardoust, S. J.; Rowshanzamir, S.; Amjadi, M. Int. J. Hydrogen Energy 2010, 35, 9349-9384.

(19) Mauritz, K. a.; Moore, R. B. Chem. Rev. 2004, 104, 4535-4585.

(20) Decker, E. R.; Levitt, D. G. Biophys. J. 1988, 53, 25-32.

(21) Heinemann, S.; Sigworth, F. J. BBA - Biomembr. 1989, 987, 814.

(22) Oiki, S.; Koeppe, R. E.; Andersen, O. S. Proc. Natl. Acad. Sci. U. S. A. 1995, 92, 2121-2125.

(23) Phillips, L. R.; Cole, C. D.; Hendershot, R. J.; Cotten, M.; Cross, T. a; Busath, D. D. Biophys. J. 1999, 77, 2492-2501.

(24) Hamada, T.; Matsunaga, S.; Yano, G.; Fusetani, N. J. Am. Chem. Soc. 2005, 127, 110-118.

(25) Oiki, S.; Muramatsu, I.; Matsunaga, S.; Fusetani, N. Nippon Yakurigaku Zasshi 1997, 110 (Suppl. 1), 195P-198P.

(26) Iwamoto, M.; Shimizu, H.; Muramatsu, I.; Oiki, S. FEBS Lett. 2010, 584, 3995-3999.

(27) Hamada, T.; Matsunaga, S.; Fujiwara, M.; Fujita, K.; Hirota, H.; Schmucki, R.; Güntert, P.; Fusetani, N. J. Am. Chem. Soc. 2010, 132, 12941-12945.

(28) Mori, T.; Kokubo, H.; Oiki, S.; Okamoto, Y. Mol. Simul. 2011, 37, 975-985.

(29) Inoue, M.; Shinohara, N.; Tanabe, S.; Takahashi, T.; Okura, K.; Itoh, H.; Mizoguchi, Y.; Iida, M.; Lee, N.; Matsuoka, S. Nat. Chem. 2010, 2, 280-285.

(30) Agmon, N. Chem. Phys. Lett. 1995, 244, 456-462.

(31) Rasaiah, J. C.; Garde, S.; Hummer, G. Annu. Rev. Phys. Chem. 2008, 59, 713-740.

(32) Cao, Z.; Peng, Y.; Yan, T.; Li, S.; Li, A.; Voth, G. A. J. Am. Chem. Soc. 2010, 132, 11395-11397.

(33) Iwamoto, M.; Matsunaga, S.; Oiki, S. Sci. Rep. 2014, 4, 3636.

(34) Cukierman, S. Biophys. J. 2000, 78, 1825-1834.

(35) Andersen, O. S. Biophys. J. 1983, 41, 147-165.

(36) Levitt, D. G.; Decker, E. R. Biophys. J. 1988, 53, 33-38.

(37) Keener, J.; Sneyd, J. Mathematical Physiology, 2nd ed.; Springer-Verlag: New York, 2009.

(38) Pomes, R.; Roux, B. 1996, 71, 19-39.

(39) Cukierman, S. Biochim. Biophys. Acta 2006, 1757, 876-885.

(40) Qin, Z.; Tepper, H. L.; Voth, G. a. J. Phys. Chem. B 2007, 111, 9931-9939.

(41) Schumaker, M. F. Front. Biosci. 2003, 8, 982-991.

(42) Oiki, S.; Iwamoto, M.; Sumikama, T. J. Gen. Physiol. 2010, 136, 363-365.

(43) Oiki, S.; Iwamoto, M.; Sumikama, T. PLoS One 2011, 6, e16578.

(44) Schumaker, M. F.; Pomès, R.; Roux, B. Biophys. J. 2000, 79, 2840-2857.

(45) Woodhull, A. M. J. Gen. Physiol. 1973, 61, 687-708.

(46) Hille, B. Ion channels of excitable membranes, 3rd ed.; Sinauer Associates Inc: Sunderland, 2001.

(47) Press, W. H.; Teukolsky, S. A.; Vetterling, W. T.; Flannery, B. P. Numerical Recipes 3rd Edition: The Art of Scientific Computing, 3rd ed.; Cambridge University Press: New York, 2007.

(48) Andersen, O. S.; Koeppe, R. E. 2nd; Roux, B. IEEE Trans Nanobioscience 2005, 4, 10-20.

(49) Becker, M. D.; Greathouse, D. V; Koeppe, R. E.; Andersen, O. S. Biochemistry 1991, 30, 8830-8839.

(50) Oiki, S.; Koeppe, R. E.; Andersen, O. S. Biophys. J. 1994, 66, 1823-1832.

(51) Iwamoto, M.; Oiki, S. Sci. Rep. 2015, 5, 9110.

(52) Barry, P. H. J. Neurosci. Methods 1994, 51, 107-116.

(53) Hall, J. E. J. genaral Physiol. 1975, 66, 531-532.

(54) Hill, T. Free Energy Transduction and Biochemical Cycle Kinetics; Dover Publications, 2004. 